\title{
ICU monitoring of ventilation distribution
}

\author{
Richard R. Mitchell, Ross M. Wilson and Diane Sierra \\ The Institute of Biomedical Engineering Sciences, Medical Research Institute, 2200 Webster Street, San \\ Francisco, CA 94115, USA
}

Keywords: ventilation distribution, respiratory monitoring, cardiac surgery

\begin{abstract}
Summary
The oxygen washin method has been shown to be a practical way to measure functional residual capacity (FRC) in the intensive care unit. The ventilator oxygen concentration is increased and measurements of respiratory flow and oxygen concentration at the mouth are made with the patient monitoring system. No additional personnel, bedside equipment or ventilator attachments are required. A feasibility study was performed to determine if this method could be used to estimate a continuous distribution of ventilation with respect to ventilation to volume ratio $\dot{V}_{\mathrm{A}} / V$. Due to gas mixing in the ventilator, the inspired oxygen fraction does not increase instantaneously to its new value. An equation was derived which models the lung as 50 discrete compartments and accounts for the transient change in mean inspired oxygen fraction. A digital computer simulation demonstrated good distribution recovery for one and two mode ventilation distributions. Continuous distributions were computed for four post cardiac surgery patients at four levels of positive end expiratory pressure (PEEP). In these patients a linear increase in the amount of ventilation in the normal $\dot{V}_{\mathrm{A}} / V$ range occurred with increasing PEEP, i.e., slow and fast spaces tended to move centrally toward a more normal $\dot{V}_{\mathrm{A}} / V$ range. At zero PEEP $26 \%$ of the ventilation occurred in the normal range and this increased to $49 \%$ at 15 PEEP. Dead space fraction was poorly estimated and spurious modes occurred in the high $\dot{V}_{\mathrm{A}} / V$ range.
\end{abstract}

\section{Introduction}

Routine monitoring of lung volume in mechanically ventilated patients may be useful for detecting the onset of atelectasis, pulmonary edema, pneumothorax and mucous plug airway obstruction. The oxygen washin method was developed to monitor FRC in the intensive care unit (ICU) [1]. Fast and slow space components were observed in normalized semilogarithmic plots of mixed expired $\mathrm{O}_{2}$ fraction following changes in the ventilator in- spired $\mathrm{O}_{2}$ fraction for ventilators with small internal mixing volumes [2]. This suggested the possibility of obtaining estimates of ventilation and volume in a two compartment model of the lung and a detailed digital computer simulation showed no significant difference between estimates of two compartment parameters using $\mathrm{O}_{2}$ washin or $\mathrm{N}_{2}$ washout data [3].

Virtually continuous distributions of ventilation $\dot{V}_{\mathrm{A}}$ with respect to ventilation to volume ratio $\dot{V}_{\mathrm{A}} / V$ have been obtained by Lewis in the Pulmonary 
Function Laboratory using $\mathrm{N}_{2}$ washout data [4]. Distributions obtained using this approach are not unique since the number of breaths analyzed is less than the number of lung compartments used in the distribution [5]. Wagner studied the 50 compartment nitrogen washout method for its information content by determining the variability of each of twelve different distributions. This study was specifically directed at the effects of nonuniqueness (not data error) on the distributions. He found that unimodal or well separated multimodal distributions with narrow modes had small variability. Broad distributions on the other hand were poorly determined. Also variability at high $\dot{V}_{\mathrm{A}} / V$ (including dead space) was large if measurements of the first few breaths were neglected.

The method presented in this paper is an extension of the above work. This extension uses the measurement of oxygen rather than nitrogen to obtain virtually continuous distributions of $\dot{V}_{\mathrm{A}}$ with respect to $\dot{V}_{\mathrm{A}} / V$. A 50 compartment lung model is used which accounts for the dynamically changing mean inspired oxygen fraction resulting from the washin of oxygen into the ventilator and gas mixing within the ventilator.

\section{Methods}

\section{Measurement system}

The $\mathrm{O}_{2}$ washin method requires measurements of oxygen fraction and respiratory flow. These are obtained from a computer-based monitoring system [6] using an Applied Electrochemistry fast response oxygen analyzer and a transparent lightweight disposable pneumotachograph [7]. The oxygen washin algorithm is requested from a keyboard at the bedside and the oxygen fraction on the ventilator is subsequently increased to 0.9 . Breathby-breath mean expired $\mathrm{O}_{2}$ fractions and the FRC estimate are updated every 30 seconds and displayed on a bedside monitor. The ventilation distributions are presently computed off-line.

\section{Mathematical model for ventilation distribution}

The model describing ventilation in the lung is identical to that used by Lewis et al. [4]. The lung is characterized by a large number of parallel compartments including dead space. The ith compartment has a fractional ventilation $\dot{V}_{i}$ and a specific ventilation $S_{i}=V_{t} \cdot \dot{V}_{i} / V_{i}$ where $V_{t}$ is the tidal volume and $V_{i}$ is the compartmental volume at the end of expiration. The oxygen washin method estimates FRC by adding the difference between mean inspired and expired oxygen fraction during steady state to each mean expired oxygen fraction and then computing the 'area' between the mean inspired and adjusted mean expired fractions. This area is proportional to FRC. The mean oxygen fraction difference during steady state is proportional to oxygen uptake and its addition to the mean expired data produces mean inspired and adjusted mean expired gas fractions which are approximately one minus the mean inspired and expired nitrogen fractions respectively. Thus, to the extent that this approximation is valid, the mean expired adjustment procedure may be used to obtain mean gas fraction data that can be treated as if it was obtained from an inert gas and continuous distributions of ventilation derived using inert gas theory.

The equation relating adjusted mean expired oxygen fraction $F_{j}$ to compartmental fractional ventilation and specific ventilation when the mean inspired oxygen fraction $F_{\mathrm{I} j}$ is varying with time is

$$
\begin{aligned}
& F_{i}=F_{0} \sum_{i=1}^{n-1} \dot{V}_{i} \omega_{j}+\sum_{i=1}^{n-1} \dot{V}_{i} S_{i} \sum_{k=1}^{j} \omega_{i}^{j-k+1} F_{\mathrm{I} k} \\
& +\dot{V}_{n} F_{\mathrm{I} j} \quad j=1, \ldots, m
\end{aligned}
$$

where $F_{0}$ is the initial inspired oxygen fraction prior to the ventilator oxygen fraction change and $\omega_{i}=$ $\left(1+S_{i}\right)^{-1}$ is the compartmental alveolar dilution ratio. Note that Eq. (1) differs from Eq. (1) in [4] by the addition of the latter two terms which account respectively for the effects of a time varying inspired gas fraction and the dead space fractional ventilation $\dot{V}_{n}$. 


\section{Estimation of ventilation distribution}

The nonnegative least squares algorithm of Lawson and Hanson is used to obtain estimates of $\dot{V}$, [8]. Forty-nine compartments with specific ventilations equally spaced on a logarithmic scale between 0.005 and 10.0 are used. The fiftieth compartment with an infinite specific ventilation is dead space. The least squares problem $A \dot{V}=F$ subject to $\dot{V}>0$ is solved where $\dot{V}$ is the fractional ventilation vector, $F$ is the adjusted expired fraction vector and $A$ is the matrix of coefficients of $\dot{V}_{i}$ in (1). The ridge regression procedure which incorporates a smoothing parameter is used to solve the nonunique least squares problem. This was introduced to the physiological literature by Evans and Wagner [9] and used by Lewis [4]. It is introduced into the least squares problem by augmenting the $F$ vector with $n$ additional zeros and row concatenating the $n \times n$ diagonal matrix $z I$ to $A$. We use $z=0.01$ which was determined by computer simulation to be a good compromise between recovered distributions containing extraneous modes ( $z$ small) and oversmoothed distributions ( $z$ large). Compartmental weighting is used which is similar to that used by Lewis [4]. The compartments are weighted by $\left[\omega_{i}\left(1-\omega_{m}^{i}\right)\right]$.

\section{Simulated ventilation distributions}

A computer simulation was developed to evaluate the methods ability to recover unimodal and bimodal distributions. The mean inspired oxygen fraction transient of a Siemens 900 B Servoventilator was fit with the sum of two exponentials. Mean expired oxygen fractions were obtained from Eq. (1) using the two exponential mean inspired oxygen fraction transient. The ventilation distribution was simulated either as one or the sum of two $\log$ normal distributions. Zero mean normally distributed measurement noise was added to both inspired and expired fractions. The standard deviation of the noise was $0.1 \% \quad \mathrm{O}_{2}$ which is approximately the variability of actual data in a relaxed patient. Three distributions were simulated - a very narrow single mode, a single mode approximating distributions seen in normal subjects and a bimodal distribution similar to that observed in some post cardiac surgery patients. A dead space fraction $V_{\mathrm{D}} / V_{\mathrm{T}}$ of 0.3 was assumed for each simulated distribution.

\section{Patient data collection protocol}

Oxygen washin measurements were obtained at 0 , 5,10 and $15 \mathrm{~cm} \mathrm{H}_{2} \mathrm{O}$ PEEP in four post cardiac surgery patients. In one patient the $15 \mathrm{~cm} \mathrm{H}_{2} \mathrm{O}$ PEEP level was not performed. The initial measurement was obtained as soon as possible after the patient arrived in the intensive care unit. Each additional measurement was made 20 minutes after the increase in PEEP to allow for a physiologic steady state to be achieved. Following each measurement the $\mathrm{O}_{2}$ fraction was returned to its original level. Arterial blood gases and cardiac output were monitored at each level of PEEP.

\section{Results}

Mean inspired and adjusted mean expired oxygen fractions are shown in Figure 1 for an oxygen washin procedure on a post cardiac surgery patient at $13 \mathrm{~cm} \mathrm{H}_{2} \mathrm{O}$ PEEP. From the near coincidence of the inspired and expired data 1) prior to the oxygen fraction change and 2) at the end of the study, it is evident that the adjustment procedure for mean expired oxygen fraction works well. The rapid but not instantaneous increase in mean inspired oxygen fraction shows the mixing characteristics of the ventilator. A variation in the steady state mean inspired oxygen fraction is observed in the last twenty breaths. The fact that the adjusted mean expired fraction closely tracks the mean inspired fraction during this period of time supports the conclusion that the source of the variation is the ventilator, gas blender or hospital oxygen supply and not the measurement system. The transient increase in mean inspired oxygen fraction together with the steady state variation in mean inspired oxygen fraction dictated the use of Eq. (1) which accounts for changes in inspired oxygen fraction. 


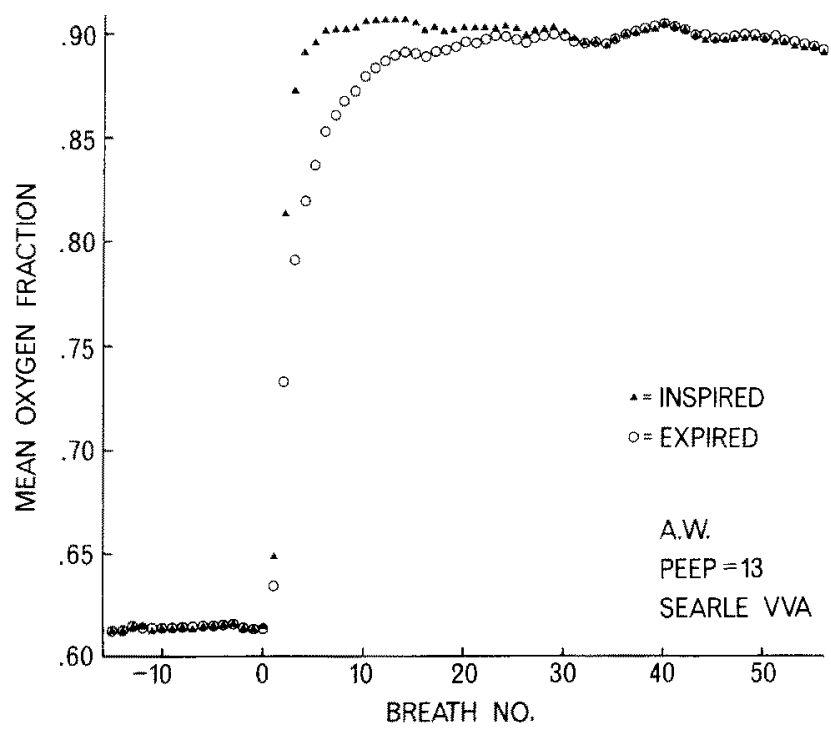

Figure 1. Oxygen washin data. Mean inspired and adjusted mean expired (see text) oxygen fractions for an oxygen washin from approximately $60 \%$ to $90 \%$. Note the rapid but not instantaneous increase in mean inspired oxygen fractions. Evidence of variation in the steady state inspired oxygen level is seen over the last 20 breaths.

\section{Recovery of simulated ventilation distributions}

The recovery of three simulated distributions is shown in Figure 2. The dead space fraction is 0.3 for all cases and is accurately estimated in each case. The location of the narrow mode in Fig. 2-A is well determined. The height of the recovered distribution is significantly less than the simulated distribution but the 0.7 fractional ventilation contained in the single mode is accurately estimated (0.69). The recovered distribution simulating a normal subject, Figure 2-B, accurately estimates the simulated mode location, shape and 0.7 modal fractional ventilation $(0.68)$. The location of the simulated mode at $S=0.2$ is accurately determined for the bimodal distribution - Figure 2-C. The second mode is recovered at a higher specific ventilation than the simulated mode at $S=1.2$. The fractional ventilations in each mode are reasonably well estimated with actual and estimated fractional ventilations being $(0.50,0.54)$ and $(0.20,0.17)$ for the low and high $\dot{V}_{\mathrm{A}} / V$ modes respectively.
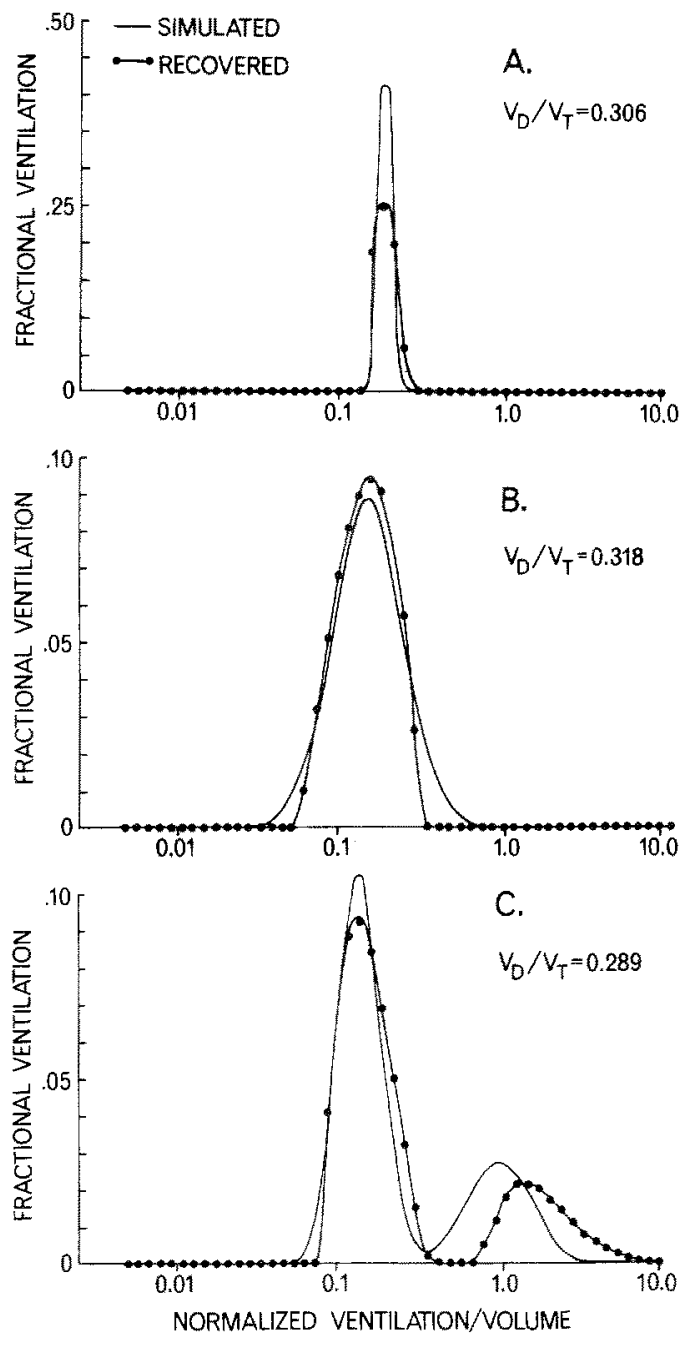

Figure 2. Recovery of simulated ventilation distributions. The ventilation distributions are generated from either a single lognormal distribution ( $A$ and $B$ ) or the sum of two lognormal distributions (C). Each lognormal distribution is characterized by the mean specific ventilation $\bar{S}$ and $\log$ standard deviation of specific ventilation $\sigma$. The fractional dead space is 0.3 in each case. The distributions are plotted with respect to specific ventilation which is a normalized ventilation to volume ratio. A. Very narrow single mode, $\bar{S}=0.2, \sigma=0.1$. B. Single mode of normal subject, $\hat{S}=0.2, \alpha=0.5$. C. Bimodal distribution of post cardiac surgery patient, $\bar{S}_{1.2}=0.15,1.2, \sigma_{1.2}=0.3,0.45$.

Ventilation distributions in post cardiac surgery patients

Ventilation distributions for a post cardiac surgery patient at four levels of PEEP are shown in Figure 3. The FRC increases linearly with PEEP from 2.1 


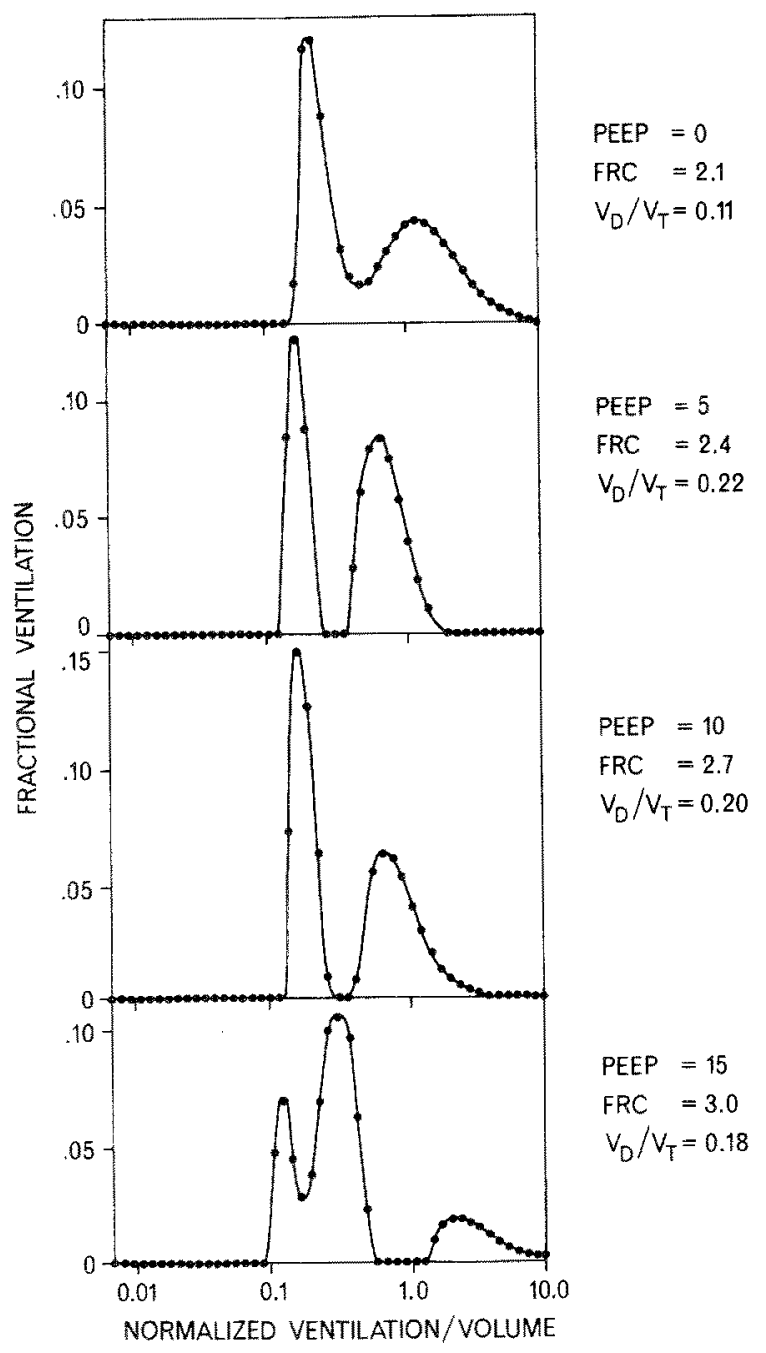

Figure 3. Distribution of ventilation in a post cardiac surgery patient at four levels of PEEP. As the FRC increases there is a leftward shift of the low $\dot{V}_{A} / V$ mode and an increase in ventilation in the normal specific ventilation range $(0.04-0.06)$.

to 3.01 . The dead space fraction increases between 0 and 5 PEEP and then remains constant. The ventilation distribution consists of two modes. The mode with a mean specific ventilation near 0.2 shifts to the left with increasing PEEP. The mode at a higher $\dot{V}_{\mathrm{A}} / V$ also shifts leftward with increasing PEEP until at $15 \mathrm{~cm} \mathrm{H}_{2} \mathrm{O}$ PEEP the two modes appear to coalesce and another high $\dot{V}_{\mathrm{A}} / V$ mode appears.

The ventilation distributions for a second post cardiac surgery patient are shown in Figure 4. Also shown is an approximate volume distribution com-

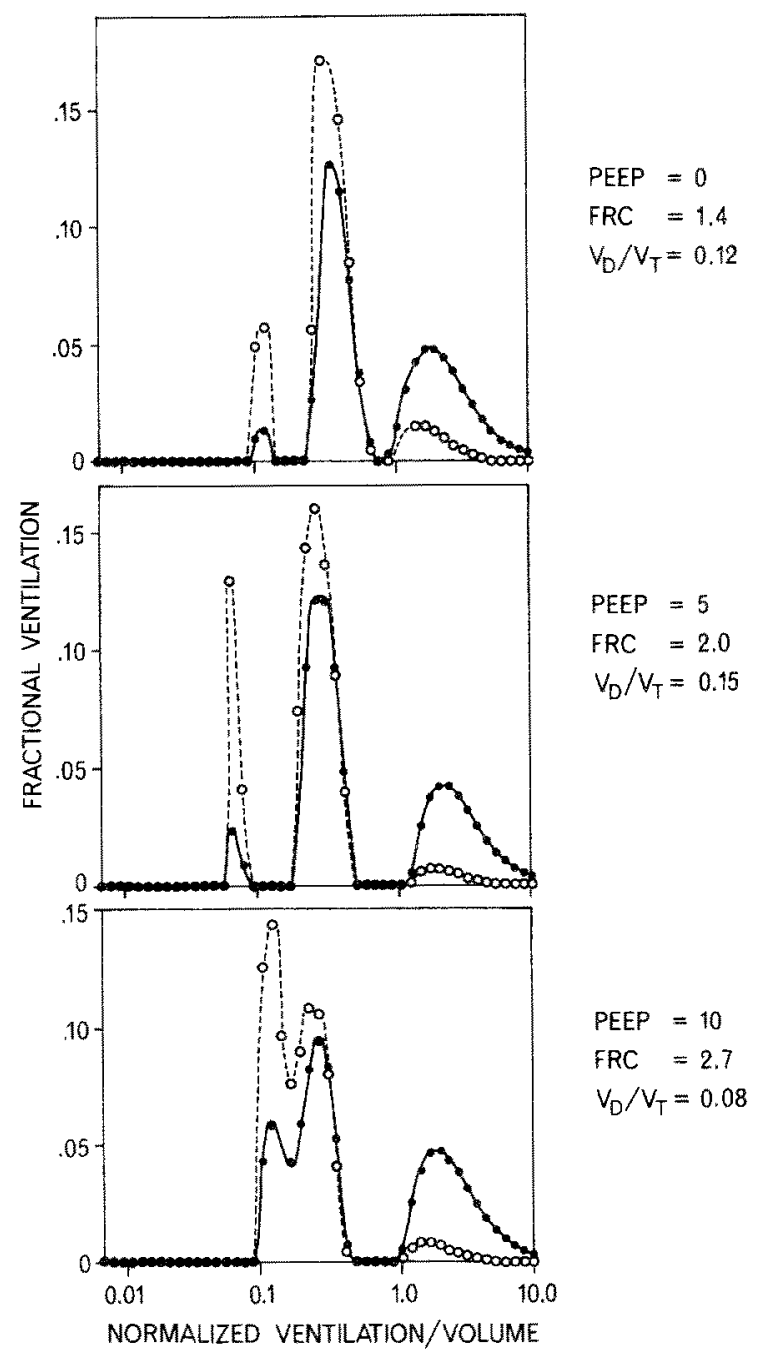

Figure 4. Distributions of ventilation $(O)$ and volume $(O)$ in a post cardiac surgery patient at three levels of PEEP. With increasing PEEP the first two modes shift leftward and then coalesce resulting in an increase in ventilation in the normal specific ventilation range $(0.04-0.06)$. The volume of the 'slow' space increases with increasing PEEP.

puted from the ventilation distribution and $\dot{V}_{\mathrm{A}} / V$ ratio. The FRC increases uniformly with PEEP. The estimate of dead space fraction remains constant with the first PEEP increase and then decreases at $10 \mathrm{~cm} \mathrm{H} \mathrm{H}_{2} \mathrm{O}$ PEEP. The ventilation and volume distributions are trimodal except at $10 \mathrm{~cm}$ $\mathrm{H}_{2} \mathrm{O}$ PEEP where the lower two $\dot{V}_{\mathrm{A}} / V$ modes coalesce. As PEEP is increased from 0 to $5 / \mathrm{cm} \mathrm{H}_{2} \mathrm{O}$, the volume of the low $\dot{V}_{\mathrm{A}} / V$ mode increases and the ventilation remains constant. The volume increase 


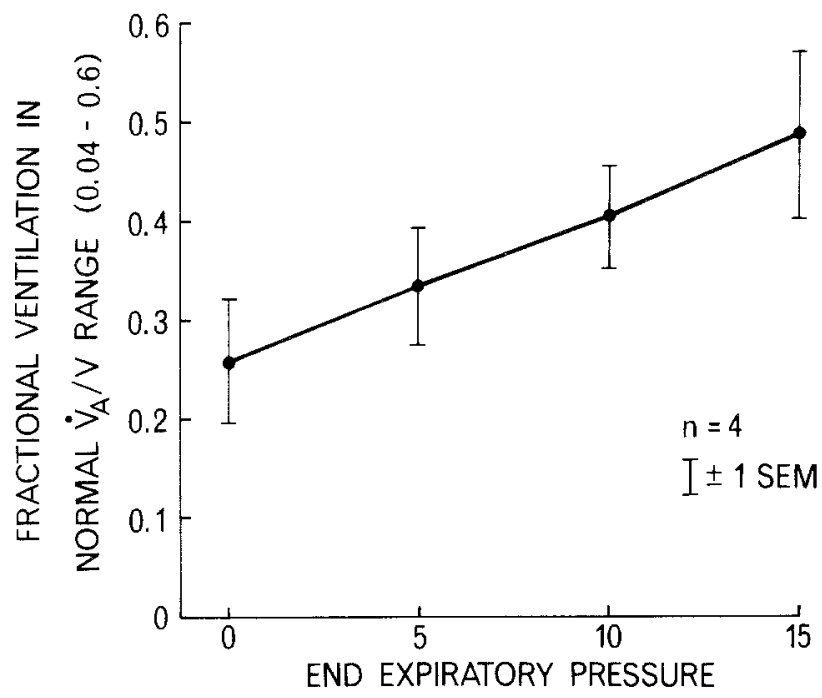

Figure 5. Fractional ventilation in the specific ventilation range 0.04 to 0.6 . This is the approximate range for eight young normal subjects found by Lewis [4]. The fractional ventilation in this range increases linearly with PEEP. Three measurements were obtained at $15 \mathrm{~cm} \mathrm{H}_{2} \mathrm{O}$ PEEP.

results in a leftward shift of the mode to a lower mean $\dot{V}_{\mathrm{A}} / V$. There is also a leftward shift of the intermediate mode with the first PEEP increase as the volume of this mode increases. With the PEEP increase from 5 to $10 \mathrm{~cm} \mathrm{H}_{2} \mathrm{O}$ the low $\dot{V}_{\mathrm{A}} / V$ mode shifts to the right, the intermediate mode shifts to the left and the two modes coalesce. The mode at high $\dot{V}_{\mathrm{A}} / V$ does not change significantly with PEEP.

The tendency for the ventilation distribution to move toward a normal $\dot{V}_{\mathrm{A}} / V$ range as PEEP is increased was observed in all four post cardiac surgery patients. Lewis studied eight young normal subjects and the approximate specific ventilation range for their distributions was 0.04 to 0.6 . The fractional ventilation in this normal specific ventilation range is shown in Figure 5 for 0 to $15 \mathrm{~cm}$ $\mathrm{H}_{2} \mathrm{O}$ PEEP. This fractional ventilation increases nearly linearly with PEEP from $26 \%$ at zero PEEP to $49 \%$ at 15 PEEP. With one exception this fraction increases for each increase in PEEP for all four patients. The majority of the variation shown by the SEM bars in Figure 5 results from differences between patients in the vertical position of the individual curves.

\section{Discussion and conclusions}

The analysis and experimental results presented in this paper represent a feasibility study to determine if continuous ventilation distributions can be obtained using the oxygen washin method in mechanically ventilated patients in the ICU. Previous experimental results and a computer simulation showed that ventilation distribution information is contained in the oxygen washin data $[2,3]$. The present computer simulation was performed to determine if ventilation distributions could be recovered from noise containing data. The simulation characterizes the mixing of gas within a mechanical ventilator and uses a unimodal or bimodal continuous ventilation distribution. Experimental data was then obtained in post cardiac surgery patients using PEEP to change the ventilation distribution of the lung. The recovered distributions were examined for expected physiological trends and any obvious artifacts.

The three simulated distributions are recovered well. The dead space fraction estimates are quite accurate with a maximum error of $6 \%$. This result was unexpected. The mixing characteristic of the ventilator results in a non-step change in inspired oxygen fraction. Thus compartments in the lung with very high $\dot{V}_{\mathrm{A}} / V$ ratios will washin almost instantaneously and will not be differentiated by the ridge regression algorithm from a compartment with an infinite $\dot{V}_{\mathrm{A}} / V$ ratio, i.e., dead space. We expected to see spurious modes representing a fraction of dead space that was erroneously recovered in a high $\dot{V}_{\mathrm{A}} / V$ range but these modes did not occur. Only three different distributions were examined and a Monte Carlo study to determine the variability of $V_{\mathrm{D}} / V_{\mathrm{T}}$ was not performed. Thus the three studies probably do not properly reflect the accuracy of the $V_{\mathrm{D}} / V_{\mathrm{T}}$ estimate and the possible existence of spurious ventilation modes at high $\dot{V}_{\mathrm{A}} / V$. The location of each single mode is determined quite well. For the two mode distribution both modes are recovered and the location of the mode at $S=0.2$ is accurately determined. The location of the mode at $S=1.2$ is overestimated, however, and this may be related to the possible poor recovery of high $\dot{V}_{\mathrm{A}} / V$ modes. For all three distributions the 
amount of ventilation in each mode is estimated very well. Although these results are only preliminary, and a Monte Carlo study must be performed, they suggest that it is possible to obtain useful ventilation distributions in patients ventilated with a Siemens 900 B Servoventilator.

The measurement of ventilation distributions in post cardiac surgery patients at increasing levels of PEEP also indicate that the method is valid. As PEEP is increased without a change in minute ventilation leftward shifts in various ventilation modes are observed. This is expected since FRC increases with PEEP and an increase in volume with no change or only a small increase in ventilation will result in a leftward shift of a ventilation mode with respect to $\dot{V}_{\mathrm{A}} / V$. The distributions change uniformly with increasing PEEP for each patient. For example, for the distribution shown in Figure 4 a low $\dot{V}_{\mathrm{A}} / V$ mode is measured at both 0 and $5 \mathrm{~cm} \mathrm{H}_{2} \mathrm{O}$ PEEP. This mode then appears to coalesce with the next mode which is uniformly moving leftward with each PEEP increase. Similarly in Figure 3, a uniform leftward shift of the lowest mode occurs. With one exception, the fractional ventilation in the normal specific ventilation range shown in Figure 5 increases for all PEEP levels for all patients. In 8 out of 11 PEEP increases the arterial oxygen partial pressure increased. Thus the fractional ventilation in the normal specific ventilation range appears to reflect the known improvement in ventilation-perfusion distribution with PEEP.

The average dead space volume for the four cardiac surgery patients is $168 \mathrm{ml}$. This can be compared with $180 \mathrm{ml}$ for a group of clinic patients [4] and $199 \mathrm{ml}$ for a group of 105 male patients with chronic airways disease [10]. Thus the dead space volume in our study is somewhat lower than the other two patient groups. The average dead space volumes at 5,10 , and $15 \mathrm{~cm} \mathrm{H}_{2} \mathrm{O}$ PEEP increased over $0 \mathrm{~cm} \mathrm{H}_{2} \mathrm{O}$ PEEP to 182,174 and $178 \mathrm{ml}$ respectively but the increase was slight and the expected uniform increase in dead space volume did not occur. A nearly linear increase in the Fowler dead space fraction from $30 \%$ to over $50 \%$ occurred as PEEP was increased from 0 to $15 \mathrm{~cm} \mathrm{H}_{2} \mathrm{O}$ in normal dogs [11].
We have no physiological explanation for the high $\dot{V}_{\mathrm{A}} / V$ modes shown in Figures 3 and 4 . We suspect that in part they reflect the inability of the present method to resolve ventilation to dead space and high $\dot{V}_{\mathrm{A}} / V$ units. Dead space ventilation recovered both as ventilation in high $\dot{V}_{\mathrm{A}} / V$ units and in the dead space compartment may explain the low dead space volumes obtained at elevated $P E E P$ levels. Ventilation modes in regions of high $\dot{V}_{\mathrm{A}} / V$ may exist in post cardiac surgery patients at elevated PEEP levels but the lack of change of these modes with PEEP (Figure 4) suggests that they are in part artifactual.

In conclusion, it appears to be possible to obtain ventilation distributions in the ICU with the oxygen washin method. In post cardiac surgery patients uniform trends in the changes in the distributions occur with PEEP. The dead space fraction is probably underestimated and ventilation modes in regions of high $\dot{V}_{\mathrm{A}} / V$ appear to be an artifact of the method.

\section{Acknowledgements}

The authors would like to thank Dr Francois Feihl for his helpful comments and analysis of this method and Ms Diane Gee for preparation of this manuscript.

This work was supported, in part, by a National Institutes of Health Grant HL-21518.

\section{References}

1. Mitchell RR, Wilson RM, Holzapfel $L$, Benis AM, Sierra $\mathrm{D}$, Osborn JJ: The oxygen washin method for monitoring functional residual capacity. Submitted to Critical Care Medicine.

2. McClung DH, Mitchell RR: Monitoring of functional residual capacity in an intensive care unit. San Diego Biomed Symp Proc 15: 403, 1976.

3. Mitchell RR, McClung DH, Bagniewski AM: Measurement of ventilation distribution with a patient monitoring system. San Diego Biomed Symp Proc 17: 277, 1978.

4. Lewis SM, Evans JW, Jalowayski AA: Continuous distributions of specific ventilation recovered from inert gas washout. J Appl Physiol: Respirat Environ Exercise Physiol 44: 416-423, 1978 
5. Wagner PD: Information content of the multibreath nitrogen washout. J Appl Physiol: Respirat Environ Exercise Physiol 46: 579-587, 1979.

6. Osborn JJ, Beaumont JO, Raison JCA, Abbott RP: Computation for quantitative on-line measurements in on intensive care ward. In: Stacey and Waxman, (eds) Comp Biomed Res, Vol. III. Academic Press, New York, 1969, pp 207-236.

7. Osborn JJ: A flowmeter for respiratory monitoring. Crit Care Med 6: 349, 1978.
8. Lawson CL, Hanson RJ: Solving Least Squares Problems. Prentice-Hall, New Jersey, 1974, p 304

9. Evans JW, Wagner PD: Limits on $\mathrm{Va} / \mathrm{Q}$ distribution from analysis of experimental inert gas elimination. J Appl Physiol: Respirat Environ Exercise Physiol 42: 889-898, 1977.

10. Lewis S, Martin CI: Characteristics of the washout dead space. Resp Physiol 36: 51-63, 1979.

11. Dueck R, Wagner PD, West JB: Effects of positive end experatory pressure on gas exchange in dogs with normal and edamatous lungs. Anesthesiology 47: 359-366, 1977. 